\title{
HTA in Germany: very special and specific
}

\author{
Wolfgang Greiner $\cdot$ J.-Matthias Graf von der Schulenburg
}

Published online: 20 January 2010

(C) Springer-Verlag 2010

This is a special issue of the European Journal of Health Economics in various ways. First, it contains solely health economic evaluations which have been submitted during the last year. We will continue this policy of publishing topical issues from time to time in which we bundle a couple of accepted papers dealing with the same topic or methodological approach. Second, it is the first issue of 2010 , the year in which we change from four to six issues per annum. This is necessary to enable publication of more of the outstanding high-quality papers which have been submitted to the EJHE. It reflects the increasing number of outstanding submissions in recent years. The editors are very grateful to the authors, the reviewers, and the publisher who made this success happen. Third, a citation index will be published for the EJHE for the first time in 2010 .

Germany will attract the attention of health economists around the world in 2010. The IQWIG (German Institute for Quality and Efficiency in Health Care) will turn into a full HTA agency assessing not only the benefits but also the costs of drugs and therapies. IQWiG will definitely start with a number of cost-benefit assessments in 2010 to support decision making on reimbursement of drugs for the German sickness funds. This will show how the methods for cost-benefit analysis published by the IQWiG will be

W. Greiner $(\bowtie)$

Department for Health Economics,

School of Public Health, University of Bielefeld,

Postfach 1001 31, Bielefeld, Germany

e-mail: wolfgang.greiner@uni-bielefeld.de

J.-M. G. von der Schulenburg

Centre for Health Economics, Leibniz University Hannover,

Hannover, Germany employed in practice. The reader of the EJHE will recall the controversies about the IQWiG's efficiency frontier to derive maximum reimbursement prices for innovative drugs.

The German pharmaceutical market is regulated by numerous instruments: negative lists for drugs which are not reimbursed, reference prices, and maximum prices for innovative drugs ( $\$ 139$ German Social Code Book V). To implement those regulations information about cost and benefits are needed. This is a change of emphasis for the German health care market. In the past reimbursement decisions were made solely on the basis of need and efficiency, which has been considered as the absence of wastefulness but not as a criterion to balance cost and benefit, especially of innovative and costly therapies.

In December 2009 IQWiG was commissioned by Germany's central reimbursement institution, the Federal Joint Commission (Gemeinsamer Bundesausschuss, GBA), with the first cost-benefit assessments. The first commission will be assessment of the cost and benefits of four antidepressive drugs (venlafaxin, duloxetin, bupropion, and mirtazapin) in comparison with other drugs currently reimbursed by the sickness finds. The second commission is an assessment of clopidogrel in combination with acetylsalicylic acid (ASA) for treatment of coronary heart disease (CHD) (and clopidogrel in monotherapy in peripheral arterial occlusive disease) in comparison to ASA monotherapy.

IQWiG's cost-benefit assessments can be used by the German sickness funds to cut reimbursement for drugs declared to be ineffective. But IQWiG's responsibilities are more than that. The institute was founded by the Federal Joint Committee in June 2004. The IQWiG's obligation is to analyse the benefits of new technologies (for example innovative drugs) and drugs with high significance to the 
health system (e.g. drug therapies with a high budget impact). In its first years the IQWiG focussed on pharmaceutical products for treatments of diabetes, asthma, CHD, and depression. According to the German Social Code Book (SGB V) the institute is also responsible for evidence-based guidelines for epidemiologically important diseases, for recommendations of disease-management programs, and for informing patients about quality and efficiency in health care in a language which can be understood by ordinary people. The Federal Joint Commission requires the IQWiG to assess specific technologies (for example drugs, operating procedures, or medical devices). The Federal Ministry of Health can also order an assessment, but this has not yet happened. Similar to the process in the National Institute for Health and Clinical Excellence (NICE) in England and Wales, assessments are mainly conducted by scientific research groups subcontracted by IQWiG.

The IQWiG is not financed by taxes but by a surcharge on expenditure by sickness funds. Thus, the institute is fully paid by sickness funds. About ten percent of Germans have private health insurance coverage. They do not contribute to the financing of the IQWiG. In 2008 the surcharge was $1.9061373 €$-Cent per office based physician case and $0.65 €$ per hospital case. The total budget of the IQWiG was about 15 Mill $€$ in 2009. The planned budget for 2010 is 13 Mill $€$. The number of IQWiG's employees rose from 33 in 2005 to more than 100 in 2009.

IQWiG's assessments of the benefits and cost effectiveness of health technologies are recommendations for the Federal Joint Committee in the decision process relating to reimbursement for pharmaceutical products. By law these assessments have to be reassessed from time to time and if necessary revised. There is no specific time period for the reassessment of IQWiG's reports. However, the pharmaceutical companies may apply for a revision if new scientific evidence is available. On the other hand it is not possible to sue the IQWiG for their assessment reports, because the IQWiG is a foundation of private law. The final report is published two months after its finalization by the federal joint committee.

The possibilities of stakeholders commenting on the methodology or the results of the assessment reports of IQWiG are rare in comparison with the NICE process. After assignment by the General Joint Committee and specification of the research question, IQWiG provides a proposal for the assessment ("Berichtsplan") which is published on IQWiG's Internet homepage. There are no clear time constraints for finalization of the assessment reports. After the interim report ("Vorbericht") is published, stakeholders, for example pharmaceutical companies, patient organizations, and medical scientific associations, have four weeks to submit comments. The IQWiG may also organize a meeting.
The IQWiG does not react in detail to the comments on their reports but there is an opportunity to amend the interim report before it is finalized and sent to the Federal Joint Committee. Till 200966 reports have been published by the IQWiG.

On October 19th, 2009, the IQWiG published version 1.0 of General Methods for the Assessment of the Relation of Benefits to Cost. This paper reflects the controversy about the peculiar IQWiG methods over the last two years. Although most of the method details meet internationally generally accepted standards (as required by German law), some very important issues still differ from international yardsticks and are in contradiction to economic theory. The most important differences from international practice are still the perspective of the evaluation, the efficiency frontier approach (as a substitute for the traditional ICER approach), and the generic use of cost-utility analysis.

The IQWiG methods paper is not very specific about the perspective. The law demands the perspective of the sickness funds' insurants. Obviously that perspective covers direct expenditure of the sickness funds and co-payments by the insured. It is unclear whether other costs, for example private expenditure on drugs and medical devices should also be assessed. For health-care providers and manufactures it is difficult to plan new studies, because the perspective might also include expenditure of other social insurance carriers or even indirect costs, but this will be decided by IQWiG on a case-by-case basis. The same applies to the choice of outcome measures, which are crucial for construction of an efficiency frontier.

The efficiency frontier, a concept for deciding the maximum reimbursement price for new drugs coming on the market with greater benefits and cost, has also attracted much attention outside Germany. The concept has never been used for this kind of decision, because it lacks theoretical foundation for pricing. For a long time it has been part of health economics textbooks as a means of identifying inefficient therapeutical alternatives on the market. As proposed by the IQWiG, the maximum reimbursement for innovative drugs is based on the current price structure, which is very much affected by regulatory decisions and not by the willingness to pay of society (or the insured). One consequence is that for manufacturers it is much easier to achieve a high price in a market with already high-priced competitors than in a market with low prices (and therefore obviously low innovation). These adverse incentives to invest in innovation are not addressed in the methods paper, although IQWiG admits that the willingness of society to pay might be higher than reflected by the efficiency frontier. But it is unclear how this could be implemented in the current assessment approach.

Most health economists agree that for a consistent reimbursement decision a non-indication-specific assessment is 
necessary. The QALY approach is only one option for comparing the benefits in different disease areas. The IQWiG still argues that a threshold of a maximum willingness to pay for a combined outcome measure would not be possible in Germany for legal reasons. It ignores that in no country is a threshold used like a hatchet, i.e. that reimbursement would be impossible beyond a fixed cost-benefit ratio. In contrast, thresholds are used as a signal for the demand of a higher degree of evidence and certainty. The acceptability of a cost-benefit ratio might vary with burden of disease, patient age, or lethality of the disease.

As the health economics capacity of the IQWiG is still quite limited, and as cost-benefit assessments will only be conducted after a benefit assessment, a large number of health economic assessments cannot be expected in the near future. Instead of this a growing number of health economic evaluations and quality of life studies will be conducted to prepare the German market for new reimbursement regulation. Germany is still a developing country in respect of cost-benefit analysis. That is why the IQWiG plays a major role in driving the scientific community for health economics (and hopefully vice versa).
It will be most interesting how the Joint Federal Commission and the Federal Sickness Funds' Association will use the IQWiG assessments in the future, especially how the budget impact and affordability will be weighted against acceptable cost-effectiveness. As IQWiG defines its methods without formal consent of the GBA, the future reimbursement decisions of the Federal Joint Commission will indirectly affect IQWiG methods. But, more importantly, this process will change the general emphasis of health policy in Germany, which still predominantly ignores the fact that rationing is unavoidable even in rich countries. The introduction of the DRG system in the financing of hospitals has already revolutionized the management of hospitals. The same might happen under the new regulation for the pharmaceutical sector in Germany.

The next few years will be crucial to how cost-benefit analysis will affect the pricing of pharmaceuticals and the allocation of scarce resources in the German health care system. The development of HTA in Germany remains exciting. 